\title{
Room temperature continuous wave operation of single-mode, edge-emitting photonic crystal Bragg lasers
}

\author{
Lin Zhu, Xiankai Sun, Guy A. Derose, Axel Scherer, and Amnon Yariv \\ Department of Electrical Engineering, Department of Applied Physics, California Institute of Technology, Pasadena, California 91125 \\ linz@,caltech.edu
}

\begin{abstract}
We report the first room temperature $\mathrm{CW}$ operation of two dimensional single-mode edge-emitting photonic crystal Bragg lasers. Single-mode lasing with single-lobed, diffraction limited far-fields is obtained for $100 \mu \mathrm{m}$ wide and $550 \mu \mathrm{m}$ long on-chip devices.
\end{abstract}

(C)2008 Optical Society of America

OCIS codes: (140.5960) Semiconductor lasers; (250.5300) Photonic integrated circuits

\section{Introduction}

The idea of using two dimensional distributed feedback in lasers dates back to 1973 [1]. Two dimensional distributed feedback lasers, also named as photonic crystal Bragg lasers, select the lasing mode using the Bragg reflection from a two dimensional weak index perturbation. Spatial coherence over a large area can be obtained, allowing for potential single-mode, high power operation with small beam divergence. In this letter, we report the first room temperature continuous wave $(\mathrm{CW})$ operation of two dimensional distributed feedback, single-mode, edge-emitting semiconductor lasers.

Our lasers are about $100 \mu \mathrm{m}$ wide and $550 \mu \mathrm{m}$ long. We measure the light-current (L-I) curve, spectrum, near-field and far-field of the laser. Despite the non-uniform intensity distribution in the near-field, the far-field is single-lobed and diffraction-limited. This proves that the photonic crystal Bragg structure can prevent filamentation and ensure a single-mode operation. We also demonstrate tuning of the lasing wavelength by changing the transverse lattice constant of the photonic crystal. This enables a fine wavelength tuning sensitivity (change of the lasing wavelength/ change of the lattice constant) of 0.072 . This dependence also proves that the lasing mode is selected by the photonic crystal lattice. These lasers break the limit of the index guiding for designing edge-emitting, single-mode semiconductor lasers using distributed feedback structures. This work constitutes a critical step toward broad-area, single-mode, high beam quality, high power semiconductor lasers with multiple available wavelengths.

\section{Design and fabrication of photonic crystal Bragg lasers}

(a)

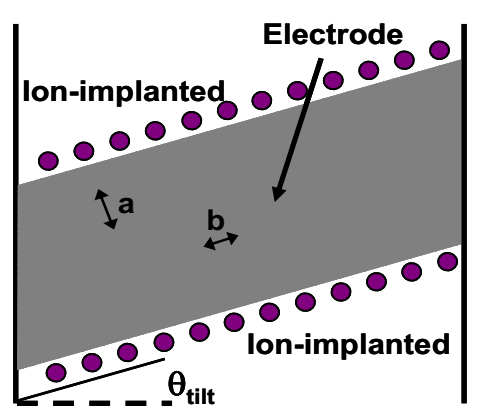

(b)

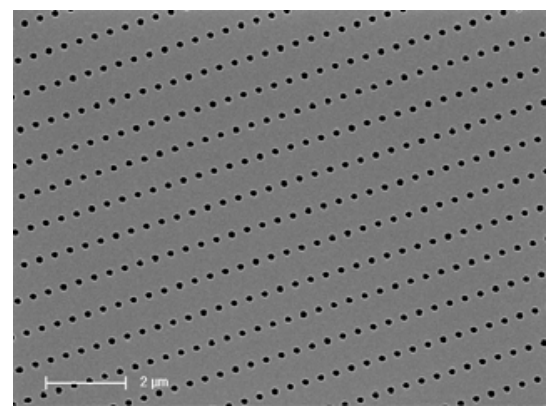

(b) SEM image of our photonic crystal lattice.

Fig.1. (a) Schematic diagram of our two dimensional photonic crystal Bragg laser.

Figure 1 shows a schematic diagram of a typical photonic crystal Bragg laser and the wafer structure. The surface photonic crystal consists of a rectangular lattice array of polymer-filled holes. The angled facets are used to suppress gain-guided modes [2-4]. In the wafer plane, an optical mode that satisfies both transverse and longitudinal Bragg resonance conditions will be confined due to the distributed Bragg reflection. Outside the metal contact region, the lasers are implanted with protons to confine the current distribution and prevent unwanted sidelobes in the far-field [5]. Figure 2 shows scanning electron microscope (SEM) images of the fabricated devices. 
We use a first order Bragg reflection for the transverse direction with a lattice constant of $a=1.04 \mu \mathrm{m}$ and a second order Bragg reflection for the longitudinal direction with a lattice constant of $b=490 \mathrm{~nm}$. The design corresponds to a resonance wavelength of $1553 \mathrm{~nm}$ (The effective index is estimated to be 3.257). The hole radius is $100 \mathrm{~nm}$ and the etch depth is about $400 \mathrm{~nm}$. The metal contact width is $100 \mu \mathrm{m}$ and the tilt angle is $13.8^{\circ}$. The lasers are cleaved to lengths of about $550 \mu \mathrm{m}$ and are $\mathrm{P}$-side up bonded to a C-mount using indium solder. The C-mount is then screwed on a thermoelectric cooling (TEC) stage and the temperature is set at $13^{\circ} \mathrm{C}$.

\section{Experiment results and discussions}
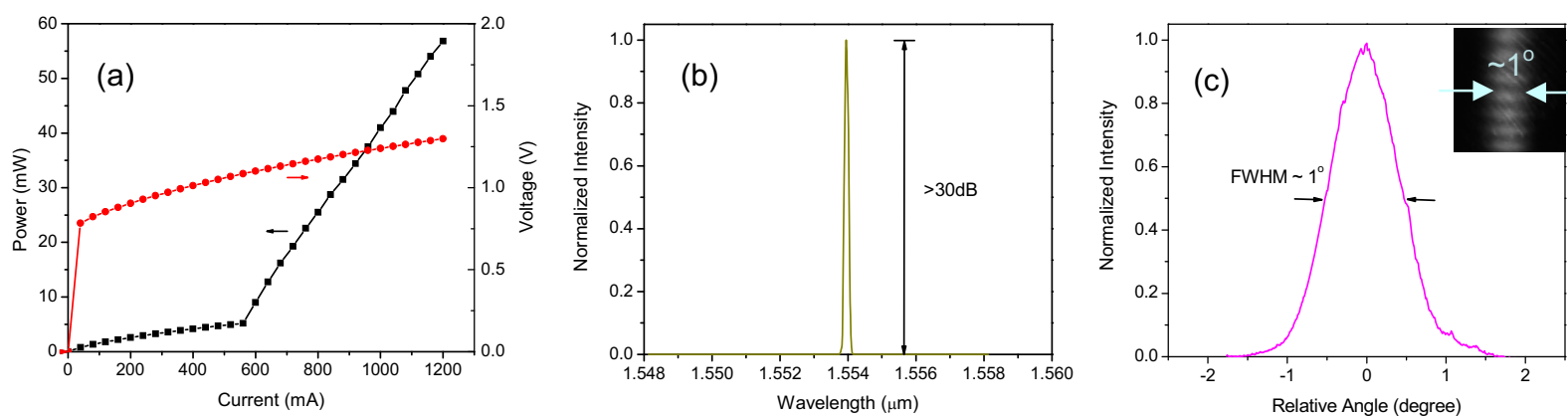

Fig.3. (a) L-I and I-V curves for the photonic crystal Bragg laser. (b) The emission spectrum at the injection current I=1.2A. (c) The far field profiles of the photonic crystal Bragg laser $(\mathrm{I}=1.2 \mathrm{~A})$. The inset is the direct image captured by the infrared CCD camera.

Single-mode CW operation of these lasers has been obtained. Figure 3a shows the photonic crystal Bragg laser characteristics of the output power versus the input current (L-I) and the voltage versus the input current (I-V). In the L-I curve, the laser has a clear threshold at $560 \mathrm{~mA}$. The device can be operated up to $\sim 2.5 \mathrm{x}$ threshold and further increase of the pumping current leads to thermal rollover. The slope efficiency is $0.08 \mathrm{~W} / \mathrm{A}$, which is similar to the broad area lasers fabricated from the same wafer under the same test conditions. This indicates that this efficiency is mainly limited by the wafer material and the thermal management. Figure $3 \mathrm{~b}$ shows the emission spectrum at an injection current of 1.2A. The single-mode operation is obtained with the side mode suppression ratio (SMSR) higher than $30 \mathrm{~dB}$. The laser also maintains the single-mode operation under different pumping currents.

The photonic crystal Bragg laser by limiting operation to a single transverse mode prevents filamentation, which leads to spatial coherence over the large emitting aperture. This results in a single-lobe diffraction-limited far field with small beam divergence. Figure $3 \mathrm{c}$ shows the far-field profile of the test laser when the pumping current is 1.2A. As shown in Fig.3c, the far-field of the photonic crystal Bragg laser is single-lobed and possesses a small beam divergence angle of $1^{\circ}$. The emitting aperture of the laser is about $100 \mu \mathrm{m}$, corresponding to a theoretical diffraction-limited far-field FWHM (full width at half maximum) width of $0.99^{\circ}$. This shows that the photonic crystal Bragg laser can operate in a single-lobe diffraction-limited far-field.

\section{Conclusion}

In summary, we have demonstrated electrically-pumped, large-area, edge-emitting photonic crystal lasers in InGaAsP active semiconductor materials operating at room temperature under $\mathrm{CW}$ condition. Single-mode lasing with single-lobed, diffraction limited far-fields is obtained for $100 \mu \mathrm{m}$ wide and $550 \mu \mathrm{m}$ long devices. These results demonstrate that photonic crystal Bragg lasers are good candidates for broad-area, single-mode, high beam quality, high power semiconductor lasers.

\section{References:}

[1] S. Wang et al, "Two-dimensional distributed-feedback lasers and their applications, ”Appl. Phys. Lett. 22, 460-462 (1973)

[2] R. J. Lang et al, "Theory of grating-confined broad-area lasers," IEEE J. Quantum Electron. 34, 2196-2210 (1998)

[3] C.S. Kim et al, "Broad-stripe near-diffraction-limited mid-infrared laser with a second-order photonic-crystal distributed feedback grating," IEEE Photon. Tech. Lett., 16, 1250-1252 (2004)

[4] L. Zhu et al, "Electrically-pumped, edge-emitting photonic crystal lasers with angled facets," Opt. Lett., 32, 1256-1258 (2007)

[5] K. Paschke,et al, "Properties of ion-implanted high-power anged-grating distributed-feedback lasers," IEEE J. Select. Topics Quantum Electron., 9, 1172-1178 (2003) 